\title{
Study on the cytotoxicity and chromosome aberration following implantation of sea coral in rabbits
}

\author{
Kannan TP, Freeda Woon Faiza Woon Tai Keat, Widowati Witjaksono and Abdul Rani Samsudin \\ School of Dental Sciences, University Sains Malaysia \\ Kubang Kerian, Kelantan - Malaysia
}

\begin{abstract}
Coral has been used as a bone substitute in many experimental studies. It has been proven to be biocompatible, biodegradable and easy to handle: and it has not been found to cause any inflammatory responses. The present study was undertaken to determine the cytotoxicity in terms of mitotic index as well as the clastogenic effect (chromosome aberration) of sea coral implantation in rabbits. The animals comprised of five male adult healthy New Zealand White (Oryctolagus cuniculus) rabbits. The biomaterial, sea coral granules used in this study was obtained from Porites species and processed by the tissue bank of Universiti Sains Malaysia, Health Campus, Kubang Kerian, Malaysia. The blood samples were collected twice from the rabbits, once before the implantation of the sea coral granules (which acted as the control) and the other, one week after the implantation (which acted as the treatment) and lymphocyte cultures were set up. The cultures were then harvested and the chromosomes were prepared for analysis. The diploid number of chromosomes in the rabbits (Oryctolagus cuniculus) was found to be 44 . Mean mitotic indices of $3.84 \pm 0.54$ per cent and $3.76 \pm 0.23$ per cent were obtained before and after implantation of sea coral granules respectively. There were no structural or numerical chromosomal aberrations observed in both the cases. The mitotic index values and chromosomal analyses in this preliminary study carried out indicate that the biomaterial, sea coral granules is non-cytotoxic and non-clastogenic under the present test conditions.
\end{abstract}

Key words: sea coral, cytotoxicity, chromosome aberration

Correspondence: Kannan TP, School of Dental Sciences, University Sains Malaysia, 16150 Kubang Kerian, Kelantan, Malaysia. Telp: 60-9-7663684 E-mail: tpkannan@kb.usm.my

\section{INTRODUCTION}

Autogenous bone graft has been considered the gold standard for bone repairing procedures as it contains triggering ingredients necessary for bone formation in bone defect. However, the availability of the autograft bone is limited and the harvesting of autografts bone causes morbidity at the donor site. Therefore, allografts are used as an alternative to autografts. Eventually, alternative bone substitute materials or biomaterials have been developed. ${ }^{1}$

Most corals are colonial organisms consisting of thousands of individual polyps. ${ }^{2}$ Natural coral graft substitutes are derived from the exoskeleton of marine madreporic corals. Coral skeletons are composed primarily of calcium carbonate $(99 \%)$ in the form of aragonite and the remaining $1 \%$ is composed of simple amino acids. ${ }^{3}$ Researchers first started evaluating corals as potential bone graft substitutes in the early 1970s in animals and in 1979 in humans. The structure of the commonly used coral, Porites, is similar to that of cancellous bone and its initial mechanical properties resemble those of bone. The exoskeleton of these high content calcium carbonate scaffolds has since been shown to be biocompatible, osteoconductive, and biodegradable at variable rates depending on the exoskeleton porosity, the implantation site and the species. Coral grafts act as an adequate carrier for growth factors and allow cell attachment, growth, spreading and differentiation. When applied appropriately and when selected to match the resorption rate with the bone formation rate of the implantation site, natural coral exoskeletons have been found to be impressive bone graft substitutes. ${ }^{4}$

Coral has been used as a bone substitute in many experimental studies. ${ }^{5,6}$ It has been proven to be biocompatible, biodegradable and easy to handle: and it has not been found to cause any inflammatory responses. ${ }^{7,8}$ An ideal bone substitute should be biocompatible, which means acceptance of the implant to the tissue surface. ${ }^{8}$ Recognition of an implant material as biocompatible nowadays depends on a large number of factors such as absence of cytotoxicity, mutagenicity, carcinogenicity, exclusion of allergenic properties, physical-chemical and biological inertia and its stability in its biological environment. ${ }^{9}$ The chromosome aberration test using cultured mammalian cells is one of the sensitive methods to predict mutagens and/or carcinogens and is a complementary test to the Salmonella/microsome assay. ${ }^{10}$ The aim of this study was to determine whether the implantation of sea-coral granules (obtained from the Porites species and processed by tissue bank of Universiti Sains Malaysia, Health Campus, Kubang Kerian, Malaysia) produced any cytotoxicity in terms of mitotic index and chromosome aberration in the lymphocytes of rabbits. 


\section{MATERIALS AND METHODS}

The Animal Ethics Committee of Health Campus, Universiti Sains Malaysia, approved this experimental study. Five adult male rabbits of New Zealand White breed were used in the present study. Blood samples were collected prior to (which acted as the control) and one week after (which acted as the treatment) the implantation of the biomaterial.

The coral used in this study is derived from a species of dead sea coral, Porites species that was harvested from the Malaysian coastal region. This coral was cleaned from debris and washed with distilled water, made into granular form and then chemically treated followed by freezedrying. After that this sample was triple packed and sterilized using gamma radiation.

The animals were anaesthetized using xylazine and ketamine and a cavity of size $2.5 \mathrm{~cm} \times 0.5 \mathrm{~cm} \times 0.5 \mathrm{~cm}$ was made in the femur of the rabbits using a surgical bur.

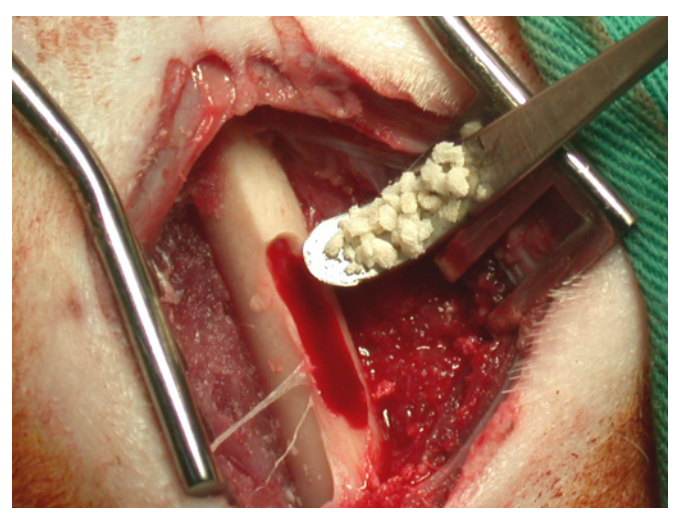

Figure 1. Implantation of sea coral granules.

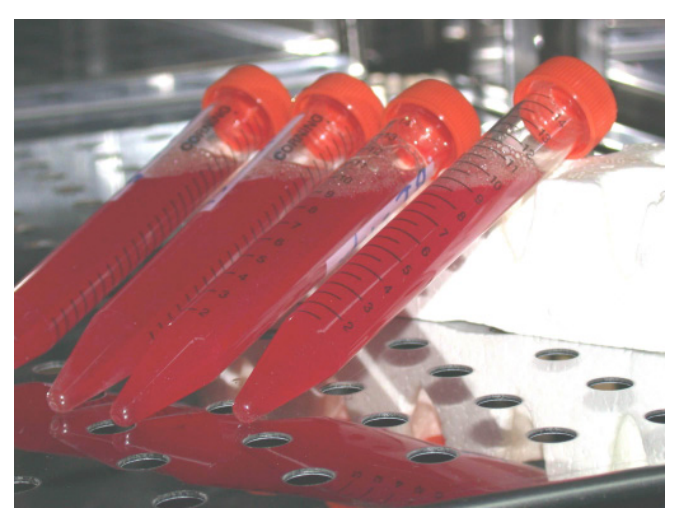

Figure 2. Incubation of culture.
$500 \mathrm{mg}$ of the biomaterial, sea coral granules was implanted in the cavity (Figure 1). Blood samples were collected from the ear vein of rabbits prior to and after the implantation of the biomaterial. RPMI 1640 medium, mitogen, phytohaemagglutinin $\mathrm{M}$, fetal bovine serum, L-glutamine, antibiotic solutions, Penicillin and streptomycin and $0.3 \mathrm{ml}$ of whole blood were the constituents of the cultures set which were incubated at $37{ }^{\circ} \mathrm{C} \mathrm{CO}_{2}$ incubator for 72 hours (Figure 2). The cultures were mixed twice daily by gentle shaking during the incubation period. Colcemid was added to the cultures $1 \frac{1}{2}$ hours prior to harvesting to arrest the cell division at metaphase. Hypotonic treatment was given using 0.56 percent potassium chloride solution for 30 minutes to swell the cells and then the cells were fixed using $3: 1$ methanol : acetic acid. The cultures were left in the refrigerator overnight at $4{ }^{\circ} \mathrm{C}$ for effective fixation of cells. Next day, the cultures were repeated with fresh fixative washes twice and the chromosomes were prepared on clean grease free slides. The slides were stained with Leishman's stain for analysis.

The mitotic index was calculated as follows.

Mitotic Index $=\frac{\text { Number of cell in metaphase }}{\text { Total number of cells counted }} \times 100$

The slides were screened for both the numerical and structural aberrations in all the samples collected prior to and after the implantation of the biomaterial.

\section{RESULTS}

Good metaphase chromosomes were obtained with RPMI 1640 as medium and the addition of colcemid $1 \frac{1}{2}$ hours prior to harvesting for culturing the lymphocytes of rabbits. The protocols of the culture like hypotonic treatment using $0.075 \mathrm{M}$ Potassium chloride solution (0.56 per cent) at $37^{\circ} \mathrm{C}$ for 30 minutes and fixation of cells overnight at $4{ }^{\circ} \mathrm{C}$ resulted in consistent results with regard to chromosome preparation. The diploid number of chromosomes in New Zealand White rabbit (Oryctolagus cuniculus) was found to be 44 .

A total of 1000 cells were counted per culture to determine the mitotic index values to assess the cytotoxicity of the sea coral granules. The mean mitotic index values of the lymphocyte cultures from the five rabbits, before and after implantation of sea coral granules were $3.84 \pm 0.54$ and $3.76 \pm 0.23$ percent respectively as given in table 1 .

Table 1. Mean mitotic indices of lymphocytes cultured from five rabbits

\begin{tabular}{cccc}
\hline \multirow{2}{*}{ Parameter } & $\mathrm{n}$ & \multicolumn{2}{c}{ Mean \pm SE } \\
\cline { 3 - 4 } & & $\begin{array}{c}\text { Before implantation of sea coral } \\
\text { granules }\end{array}$ & $\begin{array}{c}\text { After implantation of sea coral } \\
\text { granules }\end{array}$ \\
\hline Mitotic Index $(\%)$ & 1000 & $3.84 \pm 0.54$ & $3.76 \pm 0.23$ \\
\hline
\end{tabular}

$\mathrm{N}=$ number of cells counted/sample 
Two hundred metaphase spreads per culture sample were analyzed to screen for any chromosomal aberrations that might have been caused due to the implantation of sea coral granules. However, in the present study, no chromosome aberrations, either numerical or structural were observed as shown in the metaphase photos of chromosomes taken before (Figure 3 ) and after implantation of the biomaterial (Figure 4).

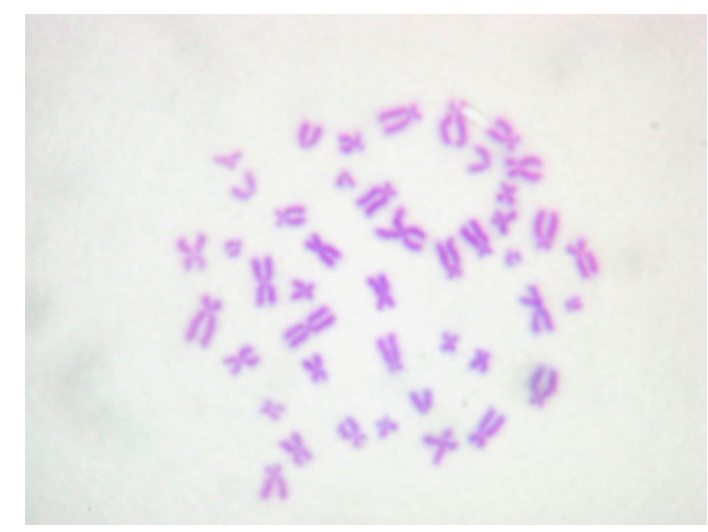

Figure 3. Metaphase spread of male rabbit (Oryctolagus cuniculus) before implantation of sea coral granules.

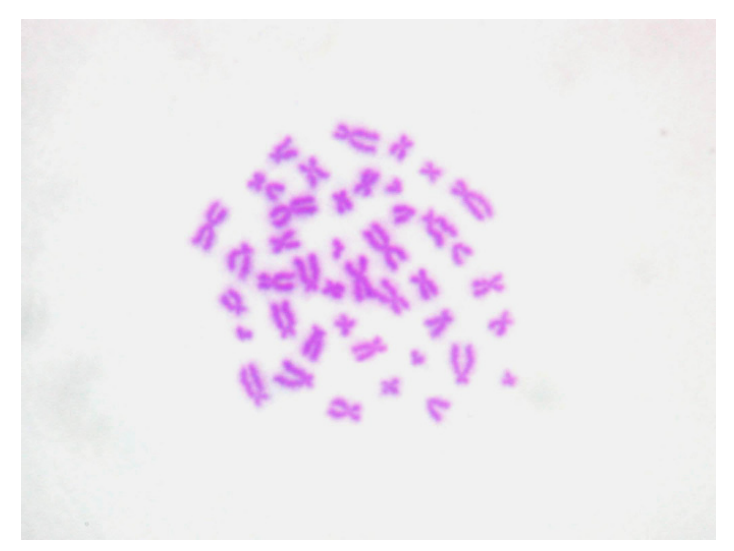

Figure 4. Metaphase spread of male rabbit (Oryctolagus cuniculus) one week after implantation of sea coral granules.

\section{DISCUSSION}

When a bone substitute with some mechanical strength is needed, hydroxyapatite appears most appropriate, as it represents the natural mineral in human bone and approximates the natural structure of cancellous bone. ${ }^{11}$ The recent developments in the use of resorptive biomaterials as osseous substitutes show a growing interest by the potential users (orthopaedic and maxillo-facial surgeons, aestheticians, etc. ${ }^{12}$ Some hydroxyapatite bone substitutes are made from bovine bone while others are produced synthetically. The sea coral granules used in the present study went through material characterization studies, biological validation studies, in vitro and in vivo and finally followed by a controlled clinical trial. This biomaterial has similar characteristics to bone with a pore size ranging from 60 to $800 \mu \mathrm{m}$. Chemical analysis using $\mathrm{X}$-ray diffraction showed that the processed coral material is composed of 99 percent calcium carbonate in the form of aragonite and was also free of other elements and toxic compounds. The current status of a new bone graft substitute is made by conversion of calcium carbonate skeleton of the coral into hydroxyapatite. Hydroxyapatite does not cause a chronic inflammatory response, toxic reactions and have excellent biocompatibility. ${ }^{13}$ In addition efforts have been made to enhance the mechanical properties of coralline hydroxyapatite to extend the field of application. ${ }^{14}$ If such materials are intended for use in medicine, the combination of two different mutagenesis studies (e.g. bacteria and mammalian cells) continues to be necessary. Many of them are based on the principle that genotoxicity of mutagenicity serves as an indicator for the carcinogenic potential of the substance. ${ }^{15}$

Under the Organization for Economic Co-operation and Development (OECD) high production volume (HPV) testing program, chromosomal aberrations tests of the HPV industrial chemicals have been conducted using Chinese hamster lung cells to examine the induction of chromosomal aberration. ${ }^{16}$ Similarly, genotoxicity of dental amalgams were assessed using chromosome aberration test in Syrian hamster embryo cells. ${ }^{17}$

The culture technique for rabbits followed in the present study for whole blood yielded consistent results which are in agreement with the reports of earlier workers. ${ }^{18-21}$ The diploid chromosome number for rabbits was reported to be $2 n=44$ first by Painter 22 in the year 1962 . The modal chromosome number of rabbit (Oryctolagus cuniculus) in the present study was also found to be $2 n=44$.

The mitotic index is a measure of cytotoxic/cytostatic effects and depends on the time after treatment. At the time of harvesting, a reduction in degree of mitotic index (greater than 50 per cent) is considered as an indication of cytotoxicity. ${ }^{23}$ A reduction greater than 50 per cent in the mitotic index value after treatment when compared to the control indicates that the biomaterial is cytotoxic in nature. However, the values of the mitotic indices obtained in the present study does not show greater than 50 per cent reduction $3.76 \pm 0.23$ per cent (after treatment) when compared to the control, $3.84 \pm 0.54$ per cent (before treatment) which indicates the non-cytotoxic nature of the biomaterial.

In this preliminary study carried out, no chromosome aberrations, either numerical or structural were observed before or after implantation of Sea coral granules. A normal complement of $2 n=44 \mathrm{XY}$ devoid of any chromosomal aberrations were observed in the cells that were analyzed, both in the control and treatment groups. This shows that the result is negative and that the biomaterial, sea coral 
granules does not cause any chromosome aberrations in cultured mammalian cells. ${ }^{23}$

The mitotic index values indicate the non-cytotoxic nature of the Sea Coral granules. Similarly, the chromosomal analyses show neither structural (clastogenicity) nor numerical aberrations (aneuploidy) in this study, which shows that the biomaterial is nonclastogenic. Hence, it can be concluded that the noncytotoxic and non-clastogenic nature of the sea coral granules may be attributed to the reason that it is devoid of any toxic compounds as well as due to the biocompatible nature of the hydroxyapatite.

\section{ACKNOWLEDGEMENTS}

The authors would like to thank Dr. Suzina Sheikh Abdul Hamid and the staff of Tissue Bank for providing the biomaterial for conducting this research. Thanks are also due to Cik. Rodiah Mohd. Radzi and En. Shaharol Anuar Abd. Latif for their help rendered in animal house during this study.

\section{REFERENCES}

1. Tuominen T, Jämsä T, Tuukkanen J, Nieminen P, Lindholm TC, Lindholm TS, Jalovaara P. Native bovine bone morphogenetic protein improves the potential of biocoral to heal segmental canine ulnar defects. International Orthopaedics 2000; 24:289-94.

2. Souter DW, Linden O. The health and future of coral reef systems. Ocean and Coastal Management 2000; 43:657-88.

3. Ouhayoun JP, Shabana AH, Issahakian S, Patat J-L, Guillemin G, Sawaf MH, Forest N. Histological evaluation of natural coral skeleton as a grafting material in miniature swine mandible. J Material Sciences 1992; 3:222-8.

4. Demers C, Hamdy CR, Corsi K, Chellat F, Tabrizian M, Yahia L. Natural coral exoskeleton as a bone graft substitute: A review. BioMedical Materials and Engineering 2002; 12:15-35.

5. Begley CT, Doherty MJ, Ram M, Wilson DJ. Comparative study of the osteoinductive properties of the bioceramic, coral and processed bone graft substitutes. Biomaterials 1995; 16:1181-95.

6. Damien CJ, Ricci JL, Christel P, Alexander H, Patat JL. Formation of a calcium phosphate rich layer on absorbable calcium carbonate bone graft substitutes. Calcif Tissue Int 1994; 55:151-8.
7. Guillemin G, Patat JL, Fournie J, Chetail M. The use of coral as a bone graft substitute. J Biomed Mater Res 1987; 21:557-67.

8. Suchanek W, Yoshimura M. Processing and properties of hydroxyapatite-based materials for use as hard tissue replacement implants. J Mater Res 1998; 13:94-103.

9. Katzer A, Marquardt H, Westendorf J, Wening JV, Von Foerster G. Polyetheretherketone-cytotoxicity and mutagenicity in vitro. Biomaterials 2002; 23:1749-59.

10. Ishidate M, Miura KF, Sofuni T. Chromosome aberration assays in genetic toxicology testing in vitro. Mutation Rresearch 1998; 404:167-72.

11. Cooke FW. Ceramics in orthopaedic surgery. Clin Orthop 1992; 276:135-46.

12. Braye F, Irigaray, Jallot E, Oudadesse H, Weber G, Deschamps N, Deschamps C, Frayssinet P, Tourenne P, Tixier H, Terver S, Lefaivre J, Amirabadi A. Resorption kinetics of osseous substitute, natural coral and synthetic hydroxyapatite. Biomaterials 1996; 17:1345-50.

13. Constantino PD, Friedman CD, Lane A. Synthetic biomaterials in facial plastic and reconstructive surgery. Facial Plastic Surgery 1993; 9:1-15.

14. Sivakumar M, Manjubala I. Preparation of hydroxyapatite/ fluoroapatite-zirconia composites using Indian corals for biomedical applications. Materials Letter 2001; 50:199-205.

15. Wiliams G. Batteries of short term tests for carcinogen screening. In: Williams G, et al., editors. The predictive value of short term screening tests in carcinogenicity evaluation. North Holland: Elsevier Biomedical Press; 1980. p. 237.

16. Kusakabe H, Yamakage K, Wakuri D, Sasaki K, Nakagawa Y, Watanabe M, Hayashi M, Sofuni T, Ono H, Tanaka N. Relevance of chemical structure and cytotoxicity to the induction of chromosome aberrations based on the testing results of 98 high production volume industrial chemicals. Mutation Research 2002; 517:187-98.

17. Akiyama M, Oshima H, Nakamura M. Genotoxicity of mercury used in chromosome aberration tests. Toxicology in vitro 2001; $15: 463-7$.

18. Stranzinger GF, Miller RC, Fechheimer NS. An improved and simple leucoctye culture technique for chromosomal preparation in rabbits. Cytologia 1974; 39:161-4.

19. Chan FPH, Sergovich FR, Shaver EL. Banding patterns in mitotic chromosomes of the rabbit (Oryctolagus Cuniculus). Can J Genet Cytol 1977; 19:625-32.

20. SchrÖder J, Suomalainen H, Van Der Loo W, SchrÖder E. Karyotypes in lymphocytes of two strains of rabbit and two species of hare. Hereditas 1978; 88:183-8.

21. SchrÖder J, Van Der Loo W. Comparison of karyotypes in three species of rabbit: Oryctolagus cuniculus, Sylvilagus nuttallii, and S. idahoensis. Hereditas 1979; 91:27-30.

22. Painter TS. Studies in mammalian spermatogenesis. VI. The chromosomes of the rabbit. J Morph Physiol 1926; 43:1-43.

23. Health Effects Test Guidelines - OPPTS 870.5375. In Vitro Chromosome Aberration Test - United States Environmental Protection Agency. 1998 August. 1-11. 